\title{
Application of Quality Control Circle in Improving the Success Rate of Indwelling Needles in Patients
}

\author{
Huiling Liu*, Baoli Heng, Xuling Li, Yahui Zhao, Huan Wang, Cuiqing Liu* \\ The First Affiliated Hospital of Jinan University, Guangzhou, China
}

\begin{abstract}
[Abstract] Objective: This study aims to assess the effectiveness of quality control circles (QCCs) in improving the success rate of intravenous indwelling needles among patients. Methods: The study included 1136 patients, that were admitted to the kidney and thoracic surgery wards of the First Affiliated Hospital of Jinan University from June 2019 to December 2019. The patients were using an indwelling needle each. They were divided into two groups: 1$)$ control group ( $n=232)$, where patients received regular nursing interventions; 2$)$ intervention group $(\mathrm{n}=904)$, where patients received QCC nursing intervention. Result: The fishbone diagram analysis revealed that poor training, lack of indwelling needle-related evaluation sheet, and lack of dynamic assessments were the factors that contributed to indwelling failure. In addition, the average indwelling duration of the intervention group was significantly higher than that of the control group. The success rate of indwelling needles in the intervention group (67.8\%) was also significantly higher than that of the control group (48.3\%), where $p<0.05$. Furthermore, the intervention group (seepage $=19.9 \%$; phlebitis $=6.7 \%$ ) reported a significantly lower prevalence of the main factors of indwelling needle failure than the control group (seepage $=34.5 \%$; phlebitis $=8.6 \%$ ), where $p<0.05$. Conclusion: The implementation of QCC can effectively reduce the occurrence of complications and nursing risks as well as improve the success rate of intravenous indwelling needles among patients.
\end{abstract}

Keyword: Quality control circle; Intravenous indwelling needle; Indwelling success rate

Publication date: May, 2021; Publication online: 31 May, 2021

*Corresponding author: Huiling Liu, Liuyxaa2021@163.com, Cuiqing Liu, CuiqingLiu@yahoo.com

\section{Introduction}

An intravenous indwelling needle is one of the most common tools for peripheral intravenous infusion ${ }^{[1]}$. Recent studies have reported that over $70 \%$ of hospitalized patients had used indwelling needles, and $69 \%$ of them experienced indwelling failure ${ }^{[2]}$. This indwelling failure was attributed to several causes: 1) indwelling needle displacement; 2) blockage of venous catheter; 3) fluid leakage; 4) oozing; 5) infection ${ }^{[3,4]}$. Removal before treatment completion causes discomfort and pain to the patient due to re-puncture. It also increases the usage of intravenous catheter during the treatment process. Moreover, in severe cases, this process may affect the duration for patients' intravenous medication. Therefore, improving the success rate of indwelling needles has become an urgent issue in clinical nursing. Recently, quality control circles (QCCs) have played important roles in improving the quality of medical treatment, and are widely applied in the field of hospital management ${ }^{[5]}$. Therefore, this study aims to evaluate the effectiveness of QCCs in improving the success rate of indwelling needles among patients.

\section{Methods}

\subsection{Participant Selection and Data Collection}

This study included 1136 patients with indwelling needles that were admitted to the kidney and thoracic surgery wards of the First Affiliated Hospital of Jinan University from June 2019 to December 2019. They were divided into a control (n $=232 ;$ males $=117$, females $=55$ ) and an intervention group $(\mathrm{n}=904$; males $=612$, females $=292)$, based on their admission date. Those admitted from June 1 to June 30, 2019, were assigned to the control group (age range: 18-83 years old) and were treated before the implementation of QCCs. Similarly, those admitted from July 1 to December 31, 2019, 
were assigned to the intervention group (age range: 12-89 years old) and treated along with the implementation of QCCs.

The inclusion criteria for participants were as follows: (1) patients who received intravenous indwelling infusion treatment in the kidney and thoracic surgery wards from June 1 to December 31, 2019; (2) patients with functional expression and communication skills; (3) patients who agreed to participate in this study. The exclusion criteria were as follows: (1) patients with mental disorders and dementia; (2) patients who underwent surgery or chemotherapy during the indwelling needle infusion.

\subsection{Regular Nursing Intervention for Patients in the Control Group}

The regular nursing invention included puncture, fixation, regular tube flushing and sealing, replacement of infusion joints and dressings, as well as infusion of drugs as per prescription by the doctors.

\subsection{QCC Nursing Intervention for Patients in the Intervention Group}

The standard QCC nursing intervention followed these steps: theme selection $\rightarrow$ activity plan implementation $\rightarrow$ status quo evaluation $\rightarrow$ goal-setting $\rightarrow$ analysis $\rightarrow$ strategy formulation $\rightarrow$ strategy implementation and review $\rightarrow$ effect confirmation $\rightarrow$ standardization $\rightarrow$ review and improvement.

\subsection{Data Analysis}

The success rate of needle indwelling at the peripheral vein was determined using the following criteria: 1) the retention time of peripheral intravenous catheter (PIVC) $\geq 72$ hours; 2 ) completion of intravenous therapy throughout the indwelling of the needle. The success rate of the indwelling needle was computed and the factors contributing to the failure of the indwelling needle was analyzed in order to determine the patients' response to the indwelling needle. A plato analysis was also performed to identify the improvement focus for QCC and set the target accordingly, while a fishbone diagram analysis was performed to identify the cause of the indwelling needle failure. Additionally, countermeasures were formulated after identifying the factors for the indwelling needle failure. These proposed countermeasures were implemented from July 1, 2019, for the patients in the intervention group.

The data collected from the intervention group were analyzed. Furthermore, the success rate of indwelling needles in the intervention group was computed to analyze the factors for indwelling failure in the control group.

Lastly, the success rate and the incidence rate of factors causing indwelling failure were compared between the intervention and the control group, respectively. The observation indicators of this study were the indwelling needle time and the factors leading to indwelling failure.

The data obtained were analyzed using Statistical Package for the Social Sciences (SPSS) version 22.0. Descriptive statistics were computed to perform t-test and chisquare tests to obtain the results.

\section{Result}

Plato induction and analysis revealed that before QCC implementation, exudation and phlebitis were the major causes for indwelling failure; thereby, being the focus points for the improvement with QCC implementation (Figure 1).

\section{Failure analysis of retention needle in control group}

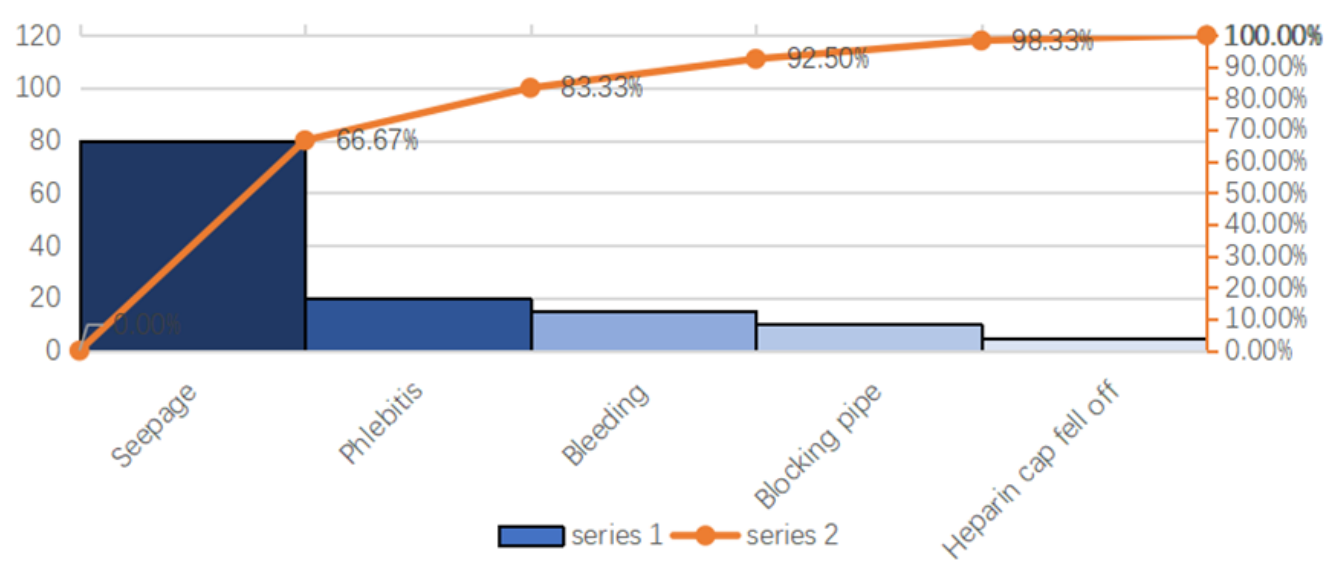

Figure 1. Analysis of the factors of indwelling failure in the control group 
The two fishbone diagrams reported that the indwelling failure could be attributed to poor training, lack of indwelling needle-related evaluation sheet, and the lack of dynamic assessments (Figure 2 and Figure 3). The diagrams also reported four main factors: 1) contractors; 2) management; 3) environment; 4) item. The analysis indicated the need for developing an indwelling needle-related evaluation sheet and an appropriate training model.

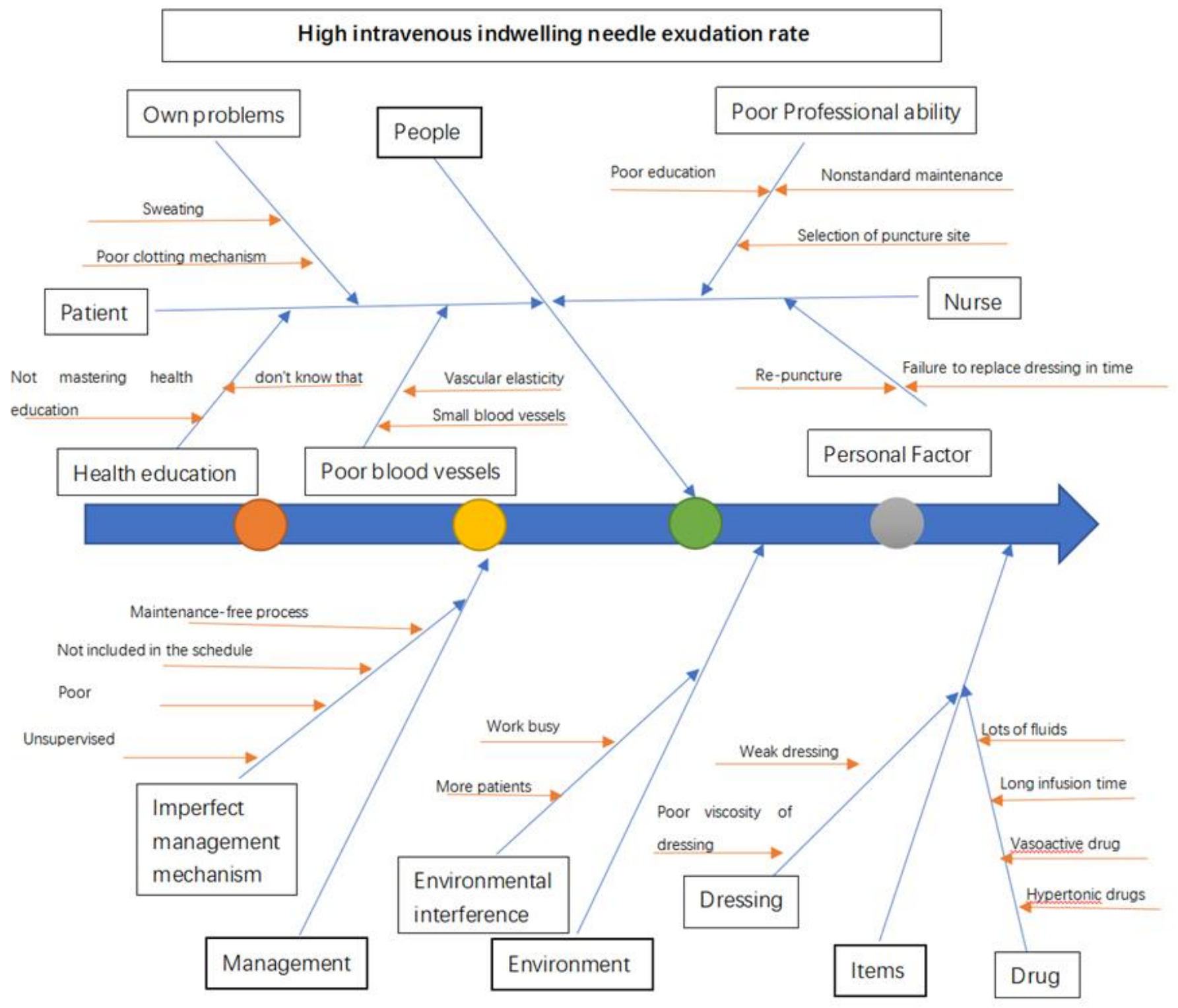

Figure 2. Fishbone diagram of the factors of indwelling failure 


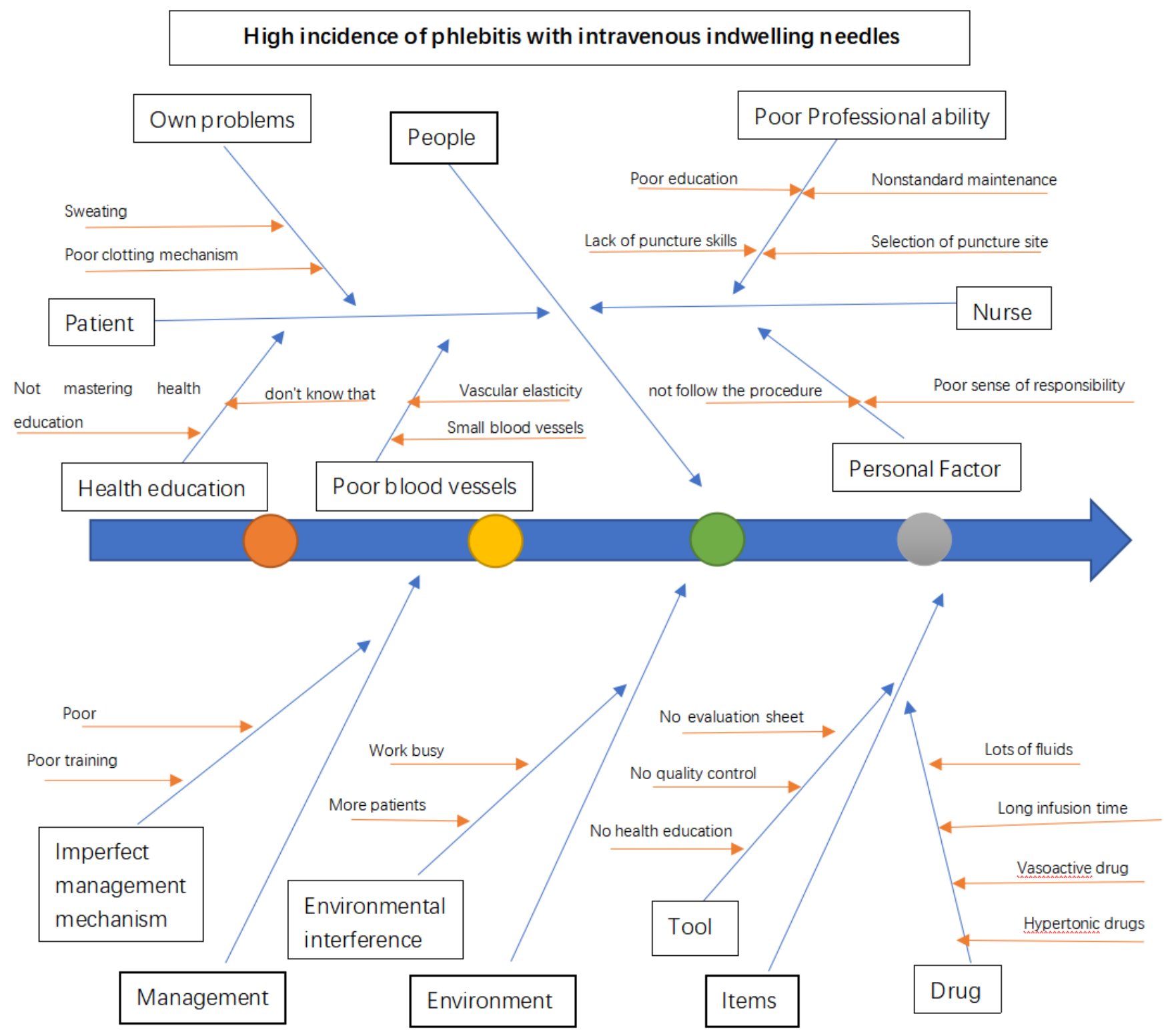

Figure 3. Fishbone diagram of the factors of indwelling failure

Table 1. Comparison of the indwelling duration between two groups of patients

\begin{tabular}{ccc}
\hline Group & Indwelling duration (hours) & Average indwelling duration (hours) \\
\hline Control group & $1.7-151.0$ & $39.7 \pm 32.2$ \\
Intervention group & $2.0-288.5$ & $64.3 \pm 43.9$ \\
\hline $\mathrm{t}$ & & -8.013 \\
$p$ value & & 0.000 \\
\hline
\end{tabular}

The longest indwelling duration was 288.5 and 151 hours for the intervention and control group, respectively. The average indwelling duration of the intervention group (64.3 \pm 43.9 hours) was significantly higher than that of the control group (39.7 \pm 32.2 hours; $p<0.05)$ (Table 1).
As shown in Table 2, the success rate of indwelling needles in the intervention group $(67.8 \% ; 613 / 904)$ was significantly higher than that of the control group (48.3\%; $112 / 232$ ), where $p<0.05$. 
Table 2. Comparison of the success rate of indwelling needles between the two groups

\begin{tabular}{ccc}
\hline Group & Indwelling success $(\mathrm{n}(\%))$ & Indwelling failure $(\mathrm{n}(\%))$ \\
\hline Control group $(\mathrm{n}=232)$ & $112(48.3)$ & $120(51.7)$ \\
Intervention group $(\mathrm{n}=904)$ & $613(67.8)$ & $291(32.2)$ \\
\hline $\mathrm{X}^{2}$ & & 30.509 \\
$p$ value & & 0.000 \\
\hline
\end{tabular}

The comparison of the main factors of indwelling failure between the two groups revealed that these factors were significantly lower in the intervention group (seepage $=$
$19.9 \%$; phlebitis $=6.7 \%$ ) than the control group (seepage $=$ $34.5 \%$; phlebitis $=8.6 \%$ ), where $p<0.05$ (Table 3 ).

Table 3. Comparison of the main factors of indwelling failure between the two groups (n (\%))

\begin{tabular}{|c|c|c|c|c|c|c|c|}
\hline Group & $\begin{array}{l}\text { Indwelling } \\
\text { success }\end{array}$ & Seepage & Phlebitis & Bleeding & Blockage & $\begin{array}{c}\text { Heparin cap } \\
\text { fell off }\end{array}$ & Other \\
\hline Control group $(\mathrm{n}=232)$ & $112(48.3)$ & $80(34.5)$ & $20(8.6)$ & $11(4.7)$ & $7(3.0)$ & $2(0.9)$ & $0(0)$ \\
\hline Intervention group $(n=904)$ & $613(67.8)$ & $180(19.9)$ & $61(6.7)$ & $9(1.0)$ & $12(1.3)$ & $5(0.5)$ & $24(2.7)$ \\
\hline$X^{2}$ & & & & & & & 53.384 \\
\hline$p$ value & & & & & & & 0.000 \\
\hline
\end{tabular}

\section{Discussion}

Intravenous indwelling needle is a widely applied nursing technique in clinical practice. This technique is effective in avoiding pain caused by repeated puncture, protecting the patients' blood vessels to a certain extent, and improving the patients' comfort level during treatment ${ }^{[1]}$. Existing research have suggested that the success rate of indwelling needles for patients benefited from the safe and effective improvement of indwelling time for patients as well as the reduction of discomfort caused by repeated punctures ${ }^{[4]}$. In this study, the effectiveness of QCCs for indwelling needle was assessed by comparing the indwelling needle duration and performing a fishbone analysis.

The findings indicated that exudation, as one of the primary influencing factors, is related to puncture technique and daily maintenance. During the puncture process, if the needle fails to enter the blood vessel in the first attempt, it needs to exit the outer wall of the blood vessel to re-enter. Despite the success of the re-entry process, this kind of indwelling needle tends to cause exudation. Currently, the puncture technique in clinics has garnered increasing attention and has been the focus of primary training for nurses; even routine standard maintenance training is considered inferior to it ${ }^{[6]}$. However, regular and standardized maintenance is extremely important to improve the success rate of indwelling needles. Therefore, in this study, a routine maintenance process for intravenous indwelling needles was designed along with a bedside shift schedule and all the nurses in the ward were trained accordingly. After implementing the bedside shift schedule, it was found that the application used for indwelling needles was loosely fitted 28 times in this study. The application was immediately replaced to prevent the detachment of the indwelling needle, provided it was noticed on time. In a study, Ota T. and other researchers reported that the catheter detachment rate was $1-26.7 \%$ due to loose application ${ }^{[7]}$.

Furthermore, a quality control schedule was developed for indwelling needles, and it was led by the head nurse or group leader every week. This schedule strengthened the supervision of indwelling needle nursing, thus improving the level of daily indwelling needle nursing.

Additionally, this study also reported that phlebitis is another major factor affecting the success rate of indwelling needles. The Visual Infusion Phlebitis scale was used to evaluate patients with phlebitis ${ }^{[8,9]}$. Most patients reported grade 1 phlebitis, where they experienced slight pain or redness around the intravenous injection site, while a few reported grade 2 phlebitis, which was characterized by pain, redness, and swelling at the injection site. There was no report 
of phlebitis more than grade 2. Phlebitis is related to the expertise of puncturing and routine maintenance, and it varies for different sites of catheterizations ${ }^{[10]}$. For example, the incidence of phlebitis for indwelling needles is lower at the forearm than at the back of the hand. Moreover, phlebitis is related to the infusion of medicine, where administration of irritant drugs and hypertonic medications, such as mannitol, can easily result in phlebitis. Therefore, chemotherapy and vasoconstrictor drugs are not suitable for intravenous indwelling catheter infusion; instead, a central venous catheter infusion should be alternatively adopted. Therefore, nurses should continuously improve their puncture expertise and routine maintenance level, in addition to practicing the selection of blood vessels with thicker outer diameter, straighter direction, and better elasticity while recognizing the forearm blood vessels as a better choice.

Despite the short study duration, an improvement rate of $40.37 \%$ was observed; thus, full participation in nursing quality management is conducive in improving performance quality and the nursing team development.

\section{Conclusion}

QCC implementation can effectively reduce the occurrence of complications and nursing risks as well as improve the success rate of intravenous indwelling needle among patients.

\section{Disclosure statement}

The authors declare that there is no conflict of interest.

\section{References}

[1] Sun L, Yang Y, 2020, Comparisons of Venous Indwelling Needles and Intravenous Infusion with Steel Needles in Pediatric Outpatient Care. Wiener Klinische Wochenschrift, 132(7-8): 205-209.

[2] Wei T, Li XY, Yue ZP, et al., 2019, Catheter Dwell Time and Risk of Catheter Failure in Adult Patients with Peripheral Venous Catheters. Journal of Clinical Nursing, 28(23-24): 4488-4495.

[3] Ying J, He J, Wu C, et al., 2020, The Preventive Effect and Safety of Personalized Nursing in Intravenous Indwelling Needle Infections. International Journal of Clinical and Experimental Medicine, 13(5): 2993-3001.

[4] Liu Y, Liu M, Han D, et al., 2019, Potential of Modified Puncture Method to Decrease Intravenous Indwelling Needle-Related Complications in Inpatients with Cardiovascular Disease. Journal of International Medical Research, 47(7): 3133-3139.

[5] Lu H, Li B, Su Q, 2018, Application of Quality Control Circle Activity in CT Quality Control Management. Zhongguo Yi Liao Qi Xie Za Zhi (Chinese Journal of Medical Instrumentation), 42(3): 232-234.

[6] Liu Y, Han D, Li X, 2018, A Modified Puncture Method Decreases the Venous Indwelling Needle Related Complications in Inpatients with Cardiovascular Diseases: A Single Center Prospective Randomized Study. Cardiology, 140: 66-66.

[7] Ota T, Tsukuda H, Tokunaga M, et al., 2015, Catheter Detachment. Oxford Medical Case Reports, (5): 292-293.

[8] Lu Y, Hao C, He W, et al., 2018, Experimental Research on Preventing Mechanical Phlebitis Arising from Indwelling Needles in Intravenous Therapy by External Application of Mirabilite. Experimental and Therapeutic Medicine, 15(1): 276-282.

[9] Tao Y, Zhou C, 2014, Risk Factors of Venous Indwelling Needle-Related Phlebitis: A Literature Review. Journal of Nursing Science, 29(20): 89-92.

[10] Xiao H, 2018, Improvement and Effect of Skin Disinfection Method in Puncture Site of Peripheral Vein Indwelling Needle. Genomics and Applied Biology, 37(11): 5180-5186. 